\title{
UNINTENDED EFFECTS OF ANONYMOUS RESUMES
}

\author{
By Luc Behaghel and Bruno Crépon and Thomas Le Barbanchon
}

\section{ONLINE APPENDIX}

* Behaghel: Paris School of Economics - Inra, 48, boulevard Jourdan, 75014 Paris, France, luc.behaghel@ens.fr. Crépon: Crest, 15 Boulevard Gabriel Peri, 92245 Malakoff, France; crepon@ensae.fr. Le Barbanchon: Crest, 15 Boulevard Gabriel Peri, 92245 Malakoff, France; tlebarbanchon@gmail.com. $\mathrm{Tel}+33623786708$. Address correspondence to Thomas Le Barbanchon. We thank participants at various conferences (SOLE 2012, ESPE 2012, ESEM 2012, EALE 2012, ASSA 2013, NORFACE 2013) and seminar participants at MIT, Columbia University, Harvard University, IZA, IAB, CREST, CEE, Cergy University, INED, and Paris School of Economics for their helpful comments. We are especially grateful to Kate Antonovics, David Autor, Marianne Bertrand, David Blau, Raj Chetty, Laurent Davezies, Larry Katz, Liam Wren-Lewis, Philip Oreopoulos, Manasa Patnam, Roland Rathelot for comments on earlier drafts of this paper. We thank the French public employment service, Pôle Emploi, for its commitment in implementing this experiment. Pôle Emploi also provided access to data and financial support for this study. We thank Andrea Lepine, Julie Moschion and Pascal Achard for excellent research assistance, Abderrazak Chebira, Yann Algan, Corinne Prost and Hélène Garner for very helpful discussion on the measurement of applicants' origin. 
Figure A-1. ExAmple of AN ANONymous Resume

$\mathrm{CV}^{\circ}$ 948311C-1-9

Technicien / Technicienne de laboratoire d'analyse industrielle

Le travail d'investigation, de contrôle conformité et mon gout pour le travail en équipe on fait que tôt je me suis orientée en chimie.

\begin{tabular}{|c|c|}
\hline \multicolumn{2}{|r|}{ EXPERIENCES PROFESSIONNELLES } \\
\hline $11 / 2008-02 / 2009$ & $\begin{array}{l}\text { Techncicienne de laboratoire, CARSO } \\
\text { rattachée au service dioxine. } \\
\text { Extraction, purification, évaporation sur échantillons d'eaux. Préparation pour analyse. }\end{array}$ \\
\hline 04/2007-06/2007 & $\begin{array}{l}\text { Technicienne qualité, Michelin } \\
\text { Stage fin de DUT. } \\
\text { Développement d'un processus de mesure et d'essai. }\end{array}$ \\
\hline \multicolumn{2}{|r|}{ FORMATION } \\
\hline 2007 & $\begin{array}{l}\text { DUT Chimie des matériaux } \\
\text { Diplôme obtenu : oui }\end{array}$ \\
\hline 2004 & $\begin{array}{l}\text { Baccalauréat STL chimie } \\
\text { Diplôme obtenu : oui }\end{array}$ \\
\hline \multicolumn{2}{|r|}{ LANGUES } \\
\hline Anglais & Très bon \\
\hline
\end{tabular}

Maitrise du Pack Office, BAFA et AFPS.

Astronomie, nouvelles technologies, voyages.

Permis : B - Véhicule léger 
Figure A-2. Signal extraction with anonymous Resumes

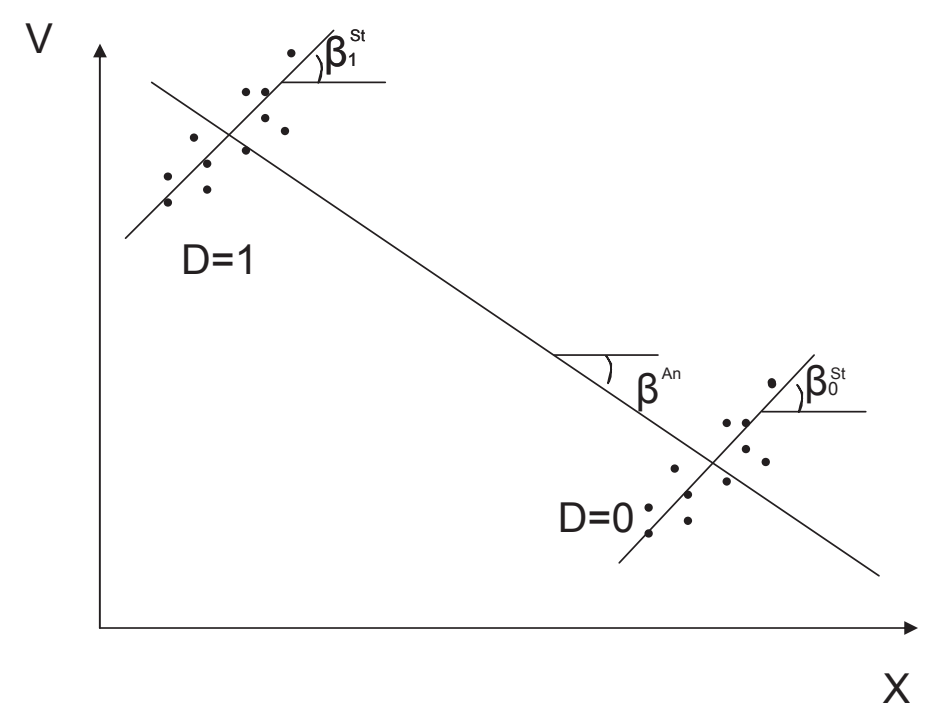

Notes: The figure illustrates signal extraction with anonymous resumes when the signal $X$ is negatively correlated with minority status $(D=1)$. Even though the resume valuation $V$ is positively correlated with $X$ conditional on $D$ (returns $\beta_{1}^{S t}>0$ and $\beta_{0}^{S t}>0$ ), the unconditional correlation is negative $\left(\beta^{A n}<0\right)$. 
Table A-1-Sample size and Response rates in the applicants' Survey for the analysis of the DECISION TO PARTICIPATE AND JOHN HENRY EFFECT

\begin{tabular}{|c|c|c|c|c|c|}
\hline & $\begin{array}{l}\text { Population } \\
\text { size (a) }\end{array}$ & $\begin{array}{c}\text { \# sampled } \\
\text { for survey }(\mathrm{b})\end{array}$ & $\begin{array}{c}\text { \# of } \\
\text { respondents (c) }\end{array}$ & $\begin{array}{l}\text { Sampling } \\
\text { rate }(b / a)\end{array}$ & $\begin{array}{l}\text { Response } \\
\text { rate }(\mathrm{c} / \mathrm{b})\end{array}$ \\
\hline \multicolumn{6}{|c|}{ A. Sample for Table 7 (applicants in firms inside and outside the experiment) } \\
\hline Participating (control firms) & 4,451 & 1,651 & 981 & 0.37 & 0.59 \\
\hline Refused to participate & 2,389 & 717 & 397 & 0.30 & 0.55 \\
\hline Total & 6,840 & 2,368 & 1,378 & 0.34 & 0.56 \\
\hline \multicolumn{6}{|c|}{ B. Sample for Table 6 (applicants in control firms with job postings after the experiment) } \\
\hline During the experiment $\square$ & 1,903 & 815 & 420 & 0.43 & 0.52 \\
\hline After the experiment & 2,548 & 836 & 387 & 0.33 & 0.46 \\
\hline Total & 4,451 & 1,651 & 807 & 0.37 & 0.49 \\
\hline
\end{tabular}

Notes:This table is restricted to firms that posted vacancies during and after the experiment. Candidates to nonparticipating firms were sampled among those firms. This explains why the population size of non-participating firms $(2,389)$ is lower than that in Table 1 although we consider candidates both during and after the experiment. Indeed only 84 non-participating firms are included here. Similarly, only control firms that also posted vacancies after the experiment (around 200) are included here. Note also that all candidates to those control firms are included (this table is not restricted to the first pool of candidate sent to the recruiter). This is why the number of candidates in control firms during the experiment $(1,903)$ is not significantly lower than that in Table 1 although this table considers fewer job vacancies. Finally, Table 6 only uses responding candidates in firms that have responding candidates during and after the experiment, so as to have a balanced panel of firms. This explains why only 807 candidates are considered as respondents (instead of 981). However, the results of Table 6 are qualitatively unchanged if one uses 981 responding candidates and an unbalanced panel of firms. 


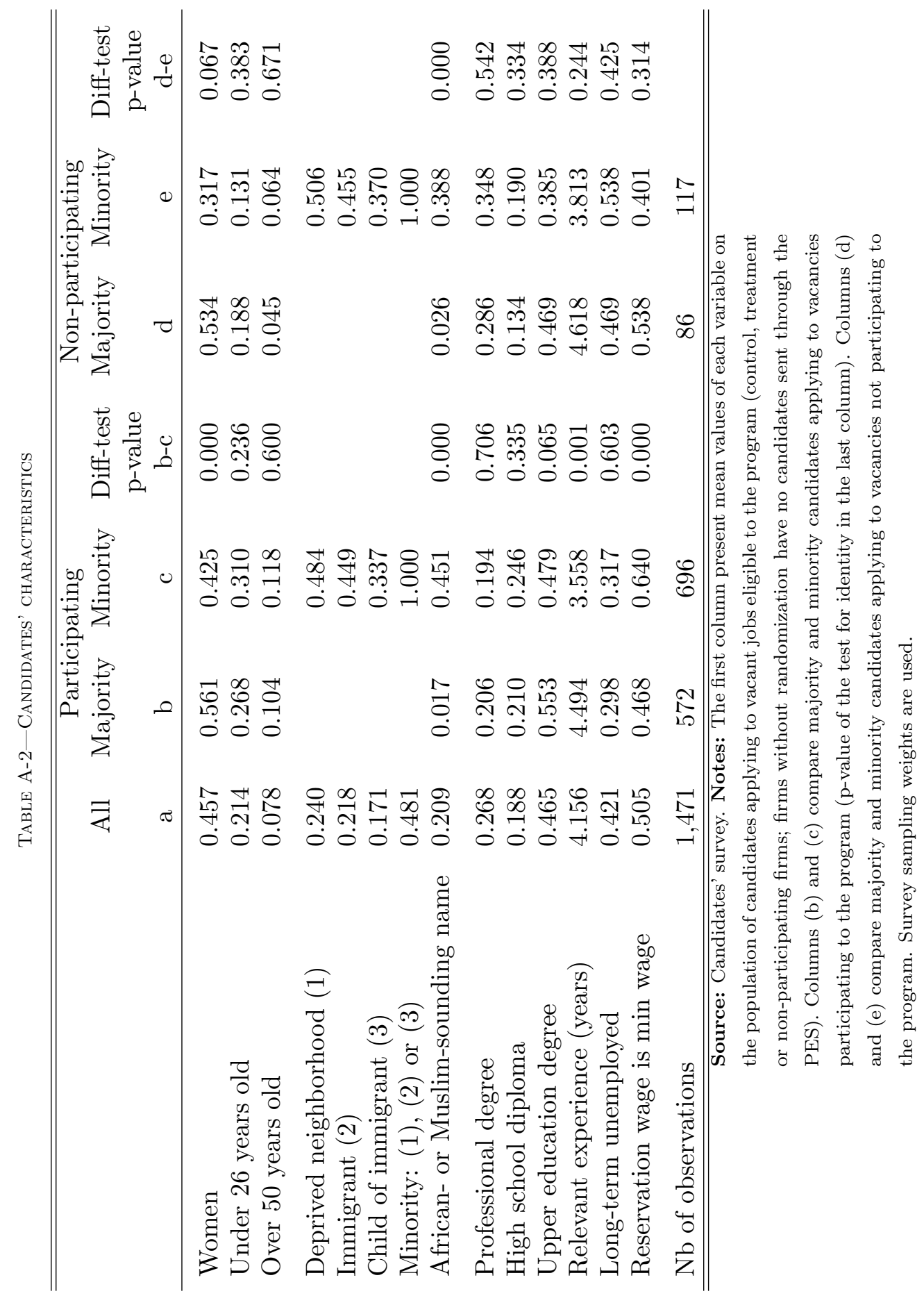




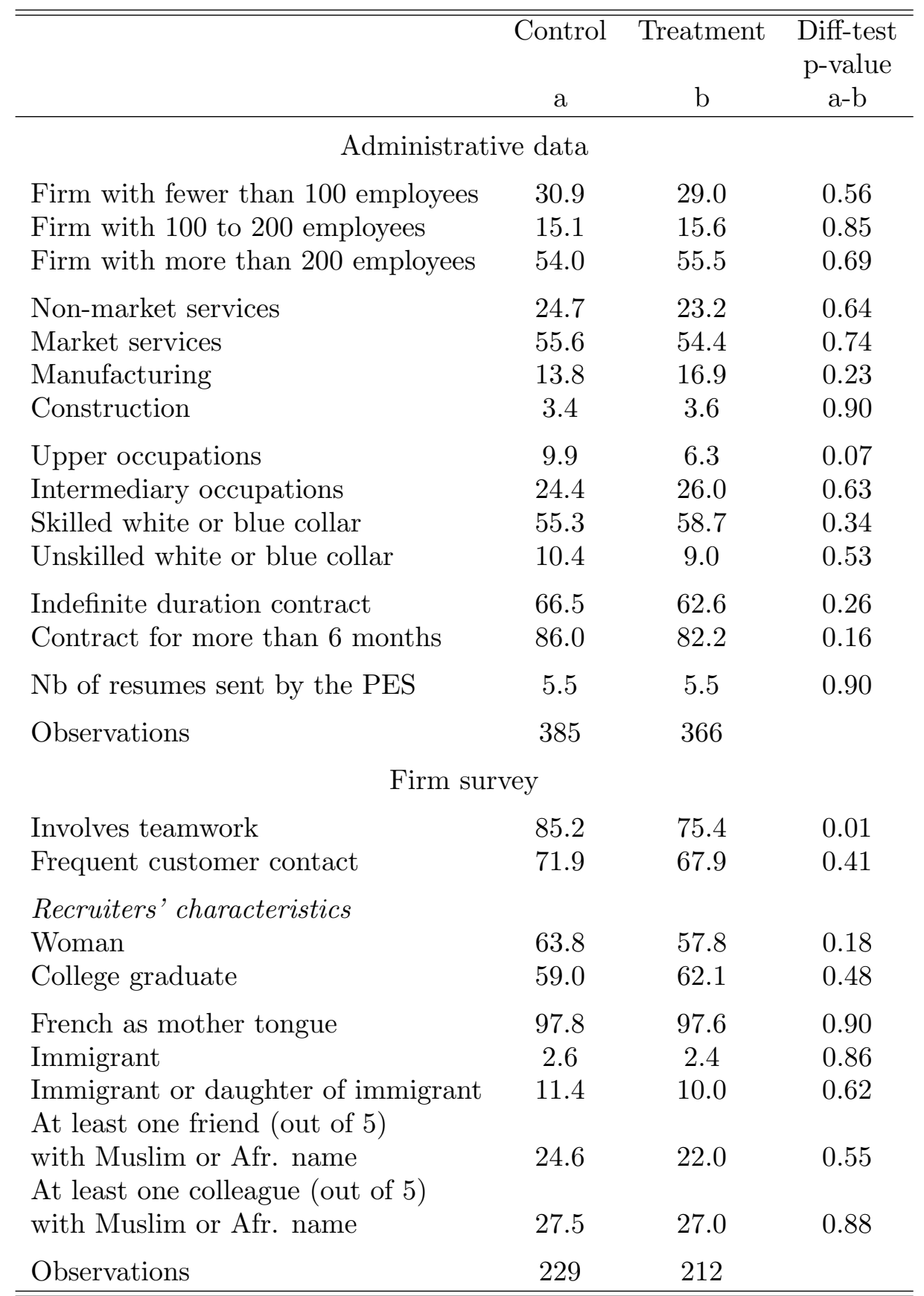

Source: PES administrative file (upper panel) and firm survey (lower panel). Notes: First three columns present mean values of each variable on the population of firms assigned to treatment and control as well as the p-value of the test of identity. 
Table A-4-Balancing test: Candidates' CharaCteristics

\begin{tabular}{|c|c|c|c|}
\hline & $\begin{array}{c}\text { Treatment } \\
\mathrm{a} \\
\end{array}$ & $\begin{array}{c}\text { Control } \\
\text { b }\end{array}$ & $\begin{array}{c}\text { Diff-test } \\
\text { p-value } \\
\text { a-b }\end{array}$ \\
\hline \multicolumn{4}{|c|}{ Candidates' Survey } \\
\hline $\begin{array}{l}\text { Women } \\
\text { Under } 26 \text { years old } \\
\text { Over } 50 \text { years old }\end{array}$ & $\begin{array}{l}0.510 \\
0.285 \\
0.130\end{array}$ & $\begin{array}{l}0.483 \\
0.291 \\
0.094\end{array}$ & $\begin{array}{l}0.580 \\
0.866 \\
0.178\end{array}$ \\
\hline $\begin{array}{l}\text { Deprived neighborhood }(1) \\
\text { Immigrant }(2) \\
\text { Child of immigrant }(3) \\
\text { Minority: (1), (2) or }(3) \\
\text { African- or Muslim-sounding name }\end{array}$ & $\begin{array}{l}0.224 \\
0.215 \\
0.169 \\
0.492 \\
0.228\end{array}$ & $\begin{array}{l}0.241 \\
0.218 \\
0.157 \\
0.474 \\
0.225\end{array}$ & $\begin{array}{l}0.557 \\
0.926 \\
0.661 \\
0.649 \\
0.922\end{array}$ \\
\hline $\begin{array}{l}\text { Professional degree } \\
\text { High school diploma } \\
\text { Upper education degree } \\
\text { Relevant experience (years) } \\
\text { Long-term unemployed } \\
\text { Reservation wage is min wage }\end{array}$ & $\begin{array}{l}0.223 \\
0.204 \\
0.521 \\
3.991 \\
0.326 \\
0.572\end{array}$ & $\begin{array}{l}0.181 \\
0.247 \\
0.514 \\
4.090 \\
0.290 \\
0.533\end{array}$ & $\begin{array}{l}0.243 \\
0.224 \\
0.873 \\
0.746 \\
0.324 \\
0.366\end{array}$ \\
\hline $\mathrm{Nb}$ of observations & 608 & 660 & \\
\hline \multicolumn{4}{|c|}{ Coding of resumes } \\
\hline $\begin{array}{l}\text { Adequate skills } \\
\text { Adequate work experience } \\
\text { Interrupted work history } \\
\text { Uncertain rating }\end{array}$ & $\begin{array}{l}0.619 \\
0.634 \\
0.336 \\
0.544\end{array}$ & $\begin{array}{l}0.480 \\
0.538 \\
0.324 \\
0.531\end{array}$ & $\begin{array}{l}0.001 \\
0.018 \\
0.751 \\
0.779\end{array}$ \\
\hline $\mathrm{Nb}$ of observations & 554 & 586 & \\
\hline
\end{tabular}




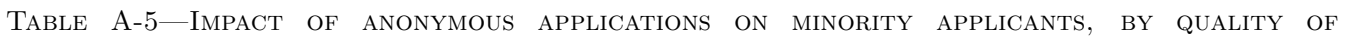
ANONYMIZATION

\begin{tabular}{|c|c|c|c|c|c|}
\hline \multirow[b]{2}{*}{ Intercept } & \multicolumn{5}{|c|}{ Interview rate } \\
\hline & $\begin{array}{c}0.094^{* * *} \\
(0.016)\end{array}$ & $\begin{array}{c}0.105^{* * *} \\
(0.020)\end{array}$ & $\begin{array}{c}0.107^{* * *} \\
(0.020)\end{array}$ & $\begin{array}{c}0.097^{* * *} \\
(0.019)\end{array}$ & $\begin{array}{c}0.110^{* * *} \\
(0.023)\end{array}$ \\
\hline Anonymous $(\mathrm{T})$ & $\begin{array}{c}-0.046^{* *} \\
(0.020)\end{array}$ & $\begin{array}{c}-0.051^{* *} \\
(0.024)\end{array}$ & $\begin{array}{l}-0.048^{*} \\
(0.025)\end{array}$ & $\begin{array}{l}-0.045^{*} \\
(0.023)\end{array}$ & $\begin{array}{l}-0.051^{*} \\
(0.028)\end{array}$ \\
\hline Has studied abroad (I) & & $\begin{array}{l}-0.053 \\
(0.039)\end{array}$ & & & \\
\hline $\mathrm{T} \times \mathrm{I}$ & & $\begin{array}{c}0.022 \\
(0.045)\end{array}$ & & & \\
\hline Has worked abroad (I) & & & $\begin{array}{l}-0.049 \\
(0.039)\end{array}$ & & \\
\hline $\mathrm{T} \times \mathrm{I}$ & & & $\begin{array}{c}0.011 \\
(0.045)\end{array}$ & & \\
\hline Speak Arabic (I) & & & & $\begin{array}{l}-0.033 \\
(0.044)\end{array}$ & \\
\hline $\mathrm{T} \times \mathrm{I}$ & & & & $\begin{array}{c}0.001 \\
(0.047)\end{array}$ & \\
\hline $\begin{array}{l}\text { Resume imperfectly } \\
\text { anonymized (Score) } \\
\text { T x Score }\end{array}$ & & & & & $\begin{array}{c}-0.043 \\
(0.035) \\
0.008 \\
(0.041)\end{array}$ \\
\hline $\begin{array}{l}\text { Vacant job controls } \\
\text { Observations }\end{array}$ & $\begin{array}{l}\text { Yes } \\
696\end{array}$ & $\begin{array}{l}\text { Yes } \\
617\end{array}$ & $\begin{array}{l}\text { Yes } \\
636\end{array}$ & $\begin{array}{l}\text { Yes } \\
649\end{array}$ & $\begin{array}{l}\text { Yes } \\
597\end{array}$ \\
\hline
\end{tabular}

Source: Candidates' survey. Notes: This table restricts the sample to minority candidates. In the first column, we estimate the average treatment effect controlling for vacant job characteristics (note that there are almost no difference with estimates in Table 4 where we do not control for any covariates). In the following columns, we introduce one-by-one some characteristics of the resumes to explore treatment effect heterogeneity. In columns 2 to 4 , the characteristics analyzed are easy to read from the resume. In the last column, we compute a score of minority status based on all signals available in the resume. When the score is over a certain threshold, the resume is said imperfectly anonymized. Survey sampling weights are used. Samples are smaller in columns 2 to 5 , because not all resumes were available as hard copy and, as already discussed, coding is thus partial. Standard errors are clustered at the vacant job level. 
TABle A-6-Robustness ANAlysis: InTERACTION BETWEen FOREIGN BACKGROUND AND RESIDENCE STATUS

\begin{tabular}{|c|c|c|c|c|}
\hline & \multicolumn{2}{|c|}{ Interview rates } & \multicolumn{2}{|c|}{ Hiring rates } \\
\hline & Baseline & Interaction & Baseline & Interaction \\
\hline & $(1)$ & $(2)$ & $(3)$ & $(4)$ \\
\hline Intercept & $\begin{array}{l}.116^{* * *} \\
(.026)\end{array}$ & $\begin{array}{l}.116^{* * *} \\
(.026)\end{array}$ & $\begin{array}{l}.021^{* *} \\
(.009)\end{array}$ & $\begin{array}{l}.021^{* *} \\
(.009)\end{array}$ \\
\hline Minority (I or D) & $\begin{array}{l}-.024 \\
(.031)\end{array}$ & & $\begin{array}{l}.002 \\
(.012)\end{array}$ & \\
\hline Immigrant (or child of) (I) & & $\begin{array}{l}-.034 \\
(.035)\end{array}$ & & $\begin{array}{l}.006 \\
(.015)\end{array}$ \\
\hline Deprived neighborhood (D) & & $\begin{array}{l}.017 \\
(.050)\end{array}$ & & $\begin{array}{l}.002 \\
(.023)\end{array}$ \\
\hline $\mathrm{I} \times \mathrm{D}$ & & $\begin{array}{l}-.019 \\
(.062)\end{array}$ & & $\begin{array}{l}-.013 \\
(.028)\end{array}$ \\
\hline Anonymous $(\mathrm{T})$ & $\begin{array}{l}.061 \\
(.040)\end{array}$ & $\begin{array}{l}.061 \\
(.040)\end{array}$ & $\begin{array}{l}.031 \\
(.019)\end{array}$ & $\begin{array}{l}.031 \\
(.019)\end{array}$ \\
\hline $\mathrm{T} \times(\mathrm{I}$ or $\mathrm{D})$ & $\begin{array}{c}-.107^{* *} \\
(.045)\end{array}$ & & $\begin{array}{l}-.037^{*} \\
(.021)\end{array}$ & \\
\hline $\mathrm{T} \times \mathrm{I}$ & & $\begin{array}{c}-.111^{* *} \\
(.048)\end{array}$ & & $\begin{array}{c}-.053^{* *} \\
(.023)\end{array}$ \\
\hline $\mathrm{T} \times \mathrm{D}$ & & $\begin{array}{c}-.148^{* *} \\
(.061)\end{array}$ & & $\begin{array}{l}-.038 \\
(.029)\end{array}$ \\
\hline $\mathrm{T} \times \mathrm{I} \times \mathrm{D}$ & & $\begin{array}{l}.197^{* *} \\
(.079)\end{array}$ & & $\begin{array}{l}.088^{* *} \\
(.042)\end{array}$ \\
\hline Nb. of obs. & 1268 & 1268 & 1268 & 1268 \\
\hline
\end{tabular}

Source: Candidates' survey. Notes: In this table, we decompose our minority indicator in its two main components: foreign background (immigrant or child of immigrant) and residence status. In column 1 (3), we recall our baseline difference-in-difference estimation for the interview (hiring) rates. In column 2 and 4, we distinguish candidates with foreign background (row 3), those residing in deprived neighborhood (row 4) and those who cumulate both (row 5). For example, in the control group, candidates with foreign background but not residing in deprived neighborhood have an interview rate that is -3.4 points lower than majority candidates. The effects of anonymization on the interview (hiring) rates of candidates with only one "discriminatory" characteristics are not statistically different whether the candidate has a foreign or lives in a deprived neighborhood: -11.1 vs. -14.8 points ( -5.3 vs. -3.8 points). OLS estimation without any covariates. Survey sampling weights are used. Standard errors are clustered at the vacant job level. 
TABle A-7-Robustness Analysis: Different measures of FOREIGN STATUS

\begin{tabular}{|c|c|c|c|c|}
\hline & $\begin{array}{l}\text { Immigrant } \\
\text { or child of } \\
\text { immigrant }\end{array}$ & Immigrant & $\begin{array}{l}\text { Child of } \\
\text { immigrant }\end{array}$ & $\begin{array}{c}\text { Muslim- or African- } \\
\text { sounding } \\
\text { names }\end{array}$ \\
\hline & \multicolumn{4}{|c|}{ Interview rates } \\
\hline \multirow[t]{2}{*}{ Intercept } & $0.116^{* * *}$ & $0.111^{* * *}$ & $0.109 * * *$ & $0.116^{* * *}$ \\
\hline & $(0.026)$ & $(0.022)$ & $(0.022)$ & $(0.023)$ \\
\hline \multirow[t]{2}{*}{ Minority (D) } & -0.024 & -0.016 & -0.013 & -0.029 \\
\hline & $(0.031)$ & $(0.030)$ & $(0.030)$ & $(0.030)$ \\
\hline \multirow[t]{2}{*}{ Anonymous (T) } & 0.061 & 0.037 & 0.037 & 0.031 \\
\hline & $(0.040)$ & $(0.034)$ & $(0.032)$ & $(0.034)$ \\
\hline \multirow[t]{2}{*}{$\mathrm{T} \times \mathrm{D}$} & $-0.107^{* *}$ & $-0.075^{*}$ & $-0.087^{* *}$ & -0.063 \\
\hline & $(0.045)$ & $(0.041)$ & $(0.040)$ & $(0.041)$ \\
\hline \multirow[t]{2}{*}{ Observations } & 1,268 & 1,268 & 1,268 & 1,268 \\
\hline & \multicolumn{4}{|c|}{ Hiring rates } \\
\hline \multirow[t]{2}{*}{ Intercept } & $0.021^{* *}$ & $0.025^{* * *}$ & $0.019^{* * *}$ & $0.022^{* * *}$ \\
\hline & $(0.009)$ & $(0.008)$ & $(0.007)$ & $(0.008)$ \\
\hline \multirow[t]{2}{*}{ Minority (D) } & 0.002 & -0.008 & 0.007 & -0.000 \\
\hline & $(0.012)$ & $(0.011)$ & $(0.013)$ & $(0.012)$ \\
\hline \multirow[t]{2}{*}{ Anonymous (T) } & 0.031 & 0.018 & 0.022 & 0.022 \\
\hline & $(0.019)$ & $(0.016)$ & $(0.015)$ & $(0.016)$ \\
\hline \multirow[t]{2}{*}{$\mathrm{T} \times \mathrm{D}$} & $-0.037^{*}$ & -0.015 & -0.027 & -0.024 \\
\hline & $(0.021)$ & $(0.019)$ & $(0.020)$ & $(0.020)$ \\
\hline Observations & 1,268 & 1,268 & 1,268 & 1,268 \\
\hline \multicolumn{5}{|c|}{$\begin{array}{l}\text { Source: Candidates' survey. Notes: Survey sampling weights are used. Standard errors are } \\
\text { clustered at the vacant job level. We verify the robustness of our analysis when we change } \\
\text { how the foreign status is measured. In the first column, we recall our baseline estimate where } \\
\text { an individual is considered foreign when he is immigrant or child of immigrant. In column } 2 \\
\text { (3), foreign status is restricted to immigrant (to child of immigrant). In column } 4 \text {, we con- } \\
\text { sider as foreigners candidates with Muslim- or African-sounding first names. In each column, } \\
\text { a candidate belongs to the minority if he has a foreign status or if he lives in a deprived } \\
\text { neighborhood. }\end{array}$} \\
\hline
\end{tabular}


TABle A-8-Robustness AnAlysis: DifFERENT SPECIFICATIONS

\begin{tabular}{|c|c|c|c|c|c|c|}
\hline & Baseline & $\begin{array}{c}\text { Basic } \\
\text { controls } \\
(2)\end{array}$ & $\begin{array}{l}\text { Controls } \\
\text { resume } \\
\text { coding } \\
(3)\end{array}$ & $\begin{array}{c}(3)+ \\
\text { vacancy } \\
\text { effects } \\
(4)\end{array}$ & $\begin{array}{c}\text { Without } \\
\text { sampling } \\
\text { weight } \\
(5)\end{array}$ & Probit \\
\hline & \multicolumn{6}{|c|}{ Interview rates } \\
\hline Intercept & $\begin{array}{c}.116^{* * *} \\
(.026)\end{array}$ & $\begin{array}{l}.137 \\
(.114)\end{array}$ & $\begin{array}{l}.157 \\
(.116)\end{array}$ & & $\begin{array}{c}.135^{* * *} \\
(.020)\end{array}$ & \\
\hline Minority & $\begin{array}{l}-.024 \\
(.031)\end{array}$ & $\begin{array}{l}-.012 \\
(.032)\end{array}$ & $\begin{array}{l}-.016 \\
(.032)\end{array}$ & $\begin{array}{l}.010 \\
(.053)\end{array}$ & $\begin{array}{l}-.028 \\
(.026)\end{array}$ & $\begin{array}{l}-.023 \\
(.030)\end{array}$ \\
\hline Anonymous (T) & $\begin{array}{l}.061 \\
(.040)\end{array}$ & $\begin{array}{l}.061 \\
(.038)\end{array}$ & $\begin{array}{l}.054 \\
(.037)\end{array}$ & & $\begin{array}{l}.058^{*} \\
(.033)\end{array}$ & $\begin{array}{l}.048 \\
(.032)\end{array}$ \\
\hline $\mathrm{T} \times$ minority & $\begin{array}{c}-.107^{* *} \\
(.045)\end{array}$ & $\begin{array}{c}-.094^{* *} \\
(.045)\end{array}$ & $\begin{array}{c}-.087^{* *} \\
(.044)\end{array}$ & $\begin{array}{l}-.096 \\
(.075)\end{array}$ & $\begin{array}{c}-.073^{*} \\
(.040)\end{array}$ & $\begin{array}{c}-.089^{* * *} \\
(.026)\end{array}$ \\
\hline \multirow[t]{2}{*}{ Nb. of obs. } & 1268 & 1268 & 1268 & 1268 & 1268 & 1268 \\
\hline & \multicolumn{6}{|c|}{ Hiring rates } \\
\hline Intercept & $\begin{array}{c}.021^{* *} \\
(.009)\end{array}$ & $\begin{array}{l}.095 \\
(.074)\end{array}$ & $\begin{array}{l}.079 \\
(.075)\end{array}$ & & $\begin{array}{c}.035^{* * *} \\
(.011)\end{array}$ & \\
\hline Minority & $\begin{array}{l}.002 \\
(.012)\end{array}$ & $\begin{array}{l}.001 \\
(.013)\end{array}$ & $\begin{array}{l}.001 \\
(.013)\end{array}$ & $\begin{array}{l}.012 \\
(.025)\end{array}$ & $\begin{array}{l}-.002 \\
(.014)\end{array}$ & $\begin{array}{l}.002 \\
(.014)\end{array}$ \\
\hline Anonymous (T) & $\begin{array}{l}.031 \\
(.019)\end{array}$ & $\begin{array}{l}.026 \\
(.018)\end{array}$ & $\begin{array}{l}.021 \\
(.018)\end{array}$ & & $\begin{array}{l}.022 \\
(.017)\end{array}$ & $\begin{array}{l}.026^{*} \\
(.016)\end{array}$ \\
\hline $\mathrm{T} \times$ minority & $\begin{array}{c}-.037^{*} \\
(.021)\end{array}$ & $\begin{array}{l}-.026 \\
(.021)\end{array}$ & $\begin{array}{l}-.024 \\
(.021)\end{array}$ & $\begin{array}{l}-.038 \\
(.038)\end{array}$ & $\begin{array}{l}-.026 \\
(.021)\end{array}$ & $\begin{array}{c}-.025^{*} \\
(.011)\end{array}$ \\
\hline Nb. of obs. & 1268 & 1268 & 1268 & 1268 & 1268 & 1268 \\
\hline
\end{tabular}

Source: Candidates' survey. Notes: The table provides different estimations of the differencein-gap equation: $Y_{i j}=\alpha_{0}+\alpha_{D} D_{i}+\alpha_{A n} A n_{j}+\delta D_{i} \times A n_{j}+\varepsilon_{i j}$. The first column considers estimation of the equation using sampling weights. It provides the reference results also reported in Table 4 The second column adds the following control variables (listed in Tables 2 and 3): firm size and industries, occupations and type of contracts offered, candidates' gender, age, education, experience, unemployment status and dummies for each 15 PES' local branches. The third column adds covariates coded on a subsample of 1,140 resumes (indicators for interrupted labor market histories, adequate work experience, adequate skills, and uncertain rating). The fourth columns adds vacancy fixed effects. The fifth uses the same specification as in the first column, but without sampling weights. The sixth column reports marginal effects of a probit estimation based on the specification of the first column. Survey sampling weights are used (except in column 5). Standard errors are clustered at the vacant job level. 
TABle A-9-Robustness ANALYSis OF FIRMS' PARTICIPATION: DIFFERENT SPECIFICATIONS

\begin{tabular}{|c|c|c|c|c|}
\hline & Baseline & $\begin{array}{c}\text { With } \\
\text { controls } \\
(2)\end{array}$ & $\begin{array}{c}\text { Without } \\
\text { sampling } \\
\text { weight } \\
(3)\end{array}$ & Logit \\
\hline \multicolumn{5}{|c|}{ Interview rates } \\
\hline Intercept & $\begin{array}{c}0.210^{* * *} \\
(0.059)\end{array}$ & $\begin{array}{c}0.200^{* * *} \\
(0.047)\end{array}$ & $\begin{array}{c}0.140^{* * *} \\
(0.028)\end{array}$ & \\
\hline Minority & $\begin{array}{c}-0.137^{* *} \\
(0.062)\end{array}$ & $\begin{array}{c}-0.115^{* *} \\
(0.049)\end{array}$ & $\begin{array}{l}-0.049 \\
(0.033)\end{array}$ & $\begin{array}{c}-0.142^{* *} \\
(0.061)\end{array}$ \\
\hline Participating $(\mathrm{P})$ & $\begin{array}{c}-0.070 \\
(0.063)\end{array}$ & $\begin{array}{l}-0.056 \\
(0.052)\end{array}$ & $\begin{array}{c}0.007 \\
(0.033)\end{array}$ & $\begin{array}{c}-0.058 \\
(0.050)\end{array}$ \\
\hline $\mathrm{P} \times$ minority & $\begin{array}{c}0.143^{* *} \\
(0.069)\end{array}$ & $\begin{array}{c}0.113^{* *} \\
(0.057)\end{array}$ & $\begin{array}{c}0.044 \\
(0.040)\end{array}$ & $\begin{array}{l}0.190^{*} \\
(0.104)\end{array}$ \\
\hline$R^{2}$ & 0.162 & 0.220 & 0.138 & \\
\hline Nb. of obs. & 1,378 & 1,378 & 1,378 & 1,378 \\
\hline \multicolumn{5}{|c|}{ Hiring rates } \\
\hline Intercept & $\begin{array}{c}0.129^{* * *} \\
(0.042)\end{array}$ & $\begin{array}{c}0.128^{* * *} \\
(0.037)\end{array}$ & $\begin{array}{c}0.101^{* * *} * \\
(0.024)\end{array}$ & \\
\hline Minority & $\begin{array}{c}-0.095^{* *} \\
(0.045)\end{array}$ & $\begin{array}{c}-0.092^{* *} \\
(0.040)\end{array}$ & $\begin{array}{c}-0.065^{* *} \\
(0.026)\end{array}$ & $\begin{array}{c}-.076^{* *} \\
(.036)\end{array}$ \\
\hline Participating $(\mathrm{P})$ & $\begin{array}{c}-0.083^{*} \\
(0.044)\end{array}$ & $\begin{array}{c}-0.078^{* *} \\
(0.037)\end{array}$ & $\begin{array}{l}-0.042 \\
(0.027)\end{array}$ & $\begin{array}{c}-.060^{* *} \\
(0.030)\end{array}$ \\
\hline $\mathrm{P} \times$ minority & $\begin{array}{l}0.087^{*} \\
(0.049)\end{array}$ & $\begin{array}{l}0.076^{*} \\
(0.040)\end{array}$ & $\begin{array}{c}0.046 \\
(0.030)\end{array}$ & $\begin{array}{c}.091 \\
(0.077)\end{array}$ \\
\hline$R^{2}$ & 0.090 & 0.136 & 0.061 & \\
\hline Nb. of obs. & 1,378 & 1,378 & 1,378 & 1,378 \\
\hline
\end{tabular}

Source: Candidates' survey. Notes: The table provides different estimations of the differencein-gap equation: $Y_{i j}=\alpha_{0}+\alpha_{D} D_{i}+\alpha_{1} P_{j}+\alpha_{2} D_{i} \times P_{j}+\epsilon_{i j}$. The first column considers estimation of the equation using sampling weights. It provides the reference results also reported in Table 7 The second column adds the whole set of variables listed in Tables 2 and 3 as control variables (firm size and industries, occupations and type of contracts offered, candidates' gender, age, education, work experience and unemployment status). The third column estimates the previous equation without sampling weights. The fourth column reports marginal effects of a logit estimation. Survey sampling weights are used (except in the third column). Standard errors are clustered at the vacant job level. 
Table A-10-: Firms' participation

Dependent variable

Mean of dep. var.

Firm with 100 to 200 employees

Firm with more than 200 employees

Non-market services

Manufacturing

Construction

Upper occupations

Intermediary occupations

Skilled white or blue collar

Indefinite duration contract

Temporary contract for more than 6 months

Involves teamwork

Frequent customer contact

Recruiters' characteristics

Woman

College graduate

French as mother tongue

Immigrant

Child of immigrant

Participation

$0.623 \quad 0.618$

(0.012) (0.022)

$-0.001 \quad 0.014$

$(0.035) \quad(0.061)$

$0.018 \quad 0.010$

(0.026) (0.045)

$-0.001 \quad-0.075$

$(0.032) \quad(0.052)$

$0.010 \quad-0.085$

$(0.042) \quad(0.069)$

$0.066 \quad 0.095$

$(0.072) \quad(0.126)$

$0.037 \quad 0.076$

$(0.054) \quad(0.083)$

$0.015 \quad-0.026$

(0.041) (0.070)

$-0.052 \quad-0.031$

$(0.037) \quad(0.064)$
-0.012

$-0.012 \quad-0.049$

$(0.031) \quad(0.046)$

$-0.048 \quad-0.057$

$(0.038) \quad(0.059)$

$-0.019$

$(0.045)$

$-0.045$

(0.039)

$-0.070^{*}$

$(0.038)$

0.067

(0.042)

$-0.032$

(0.087)

0.005

(0.110)

$-0.082$

(0.069) 
... table A-10 continued

\begin{tabular}{lcc}
\hline \hline Dependent variable & \multicolumn{2}{c}{ Participation } \\
& $(1)$ & $(2)$ \\
\hline At least one friend (out of 5) with Muslim or Afr. name & & -0.057 \\
& & $(0.050)$ \\
At least one colleague (out of 5) with Muslim or Afr. name & & 0.017 \\
& & $(0.045)$ \\
Local PES branch fixed effects & Yes & Yes \\
Observations & 1,613 & 721 \\
R-squared & 0.277 & 0.484 \\
\hline
\end{tabular}

Source: PES administrative file and firm survey. Notes: We estimate linear probability models of the firms' decision to participate. The reference group is made up of firms with fewer than 100 employees selling services in the market sector and posting a vacancy for an unskilled position. Column 1 relies on characteristics available in the administrative file (exhaustive population). In column 2 , we add covariates obtained from the firm survey (restricting the sample to respondents). Thus survey sampling weights are used in column 2. Robust standard errors are in parentheses. We compute the F-test of the joint nullity of the coefficients in column 1 (resp. in column 2) related to size 0.73 (0.97), industries $0.83(0.28)$, occupations $0.04(0.44)$ and contracts $0.39(0.52)$. In column 2, we also compute the test related to work type 0.39 and recruiters' characteristics 0.22 . 
TABle A-11-Effects of Different ElEments of the REsume on the INTERVIEW DeCision

\begin{tabular}{|c|c|c|c|c|c|}
\hline & \multicolumn{2}{|c|}{ Standard application } & \multirow{2}{*}{$\begin{array}{c}\text { Test } \\
\text { p-value } \\
\beta^{0}(S t)=\beta^{1}(S t) \\
(3)\end{array}$} & \multirow{2}{*}{$\begin{array}{c}\text { Anonymous } \\
\text { All candidates } \\
\beta(A n) \\
(4)\end{array}$} & \multirow{2}{*}{$\begin{array}{c}\text { Test } \\
\text { p-value } \\
\beta^{0}(S t)=\beta^{1}(S t)=\beta(A n) \\
(5)\end{array}$} \\
\hline & $\begin{array}{c}\text { Majority } \\
\beta^{0}(S t) \\
(1)\end{array}$ & $\begin{array}{l}\text { Minority } \\
\beta^{1}(S t) \\
(2)\end{array}$ & & & \\
\hline Minority effect $(\alpha)$ & & $\begin{array}{l}-0.014 \\
(0.090)\end{array}$ & & & \\
\hline High overall rating & $\begin{array}{c}0.078 \\
(0.066)\end{array}$ & $\begin{array}{l}0.074^{*} \\
(0.038)\end{array}$ & 0.961 & $\begin{array}{c}0.044 \\
(0.044)\end{array}$ & 0.853 \\
\hline $\begin{array}{l}\text { Has interrupted } \\
\text { work history }\end{array}$ & $\begin{array}{l}-0.090 \\
(0.060)\end{array}$ & $\begin{array}{c}0.038 \\
(0.046)\end{array}$ & $0.070^{*}$ & $\begin{array}{l}-0.044 \\
(0.040)\end{array}$ & 0.156 \\
\hline Adequate skills & $\begin{array}{c}0.120^{* *} \\
(0.056)\end{array}$ & $\begin{array}{c}0.044 \\
(0.045)\end{array}$ & 0.304 & $\begin{array}{c}0.032 \\
(0.040)\end{array}$ & 0.428 \\
\hline $\begin{array}{l}\text { Adequate work } \\
\text { experience }\end{array}$ & $\begin{array}{l}-0.056 \\
(0.059)\end{array}$ & $\begin{array}{c}0.007 \\
(0.049)\end{array}$ & 0.416 & $\begin{array}{c}0.031 \\
(0.035)\end{array}$ & 0.444 \\
\hline High uncertainty & $\begin{array}{c}0.064 \\
(0.055)\end{array}$ & $\begin{array}{c}0.076 \\
(0.047)\end{array}$ & 0.862 & $\begin{array}{c}-0.098^{* *} \\
(0.050)\end{array}$ & $0.024^{* *}$ \\
\hline $\mathrm{Nb}$ of candidates & 252 & 334 & & 554 & \\
\hline $\mathrm{Nb}$ of vacant jobs & & & & 270 & \\
\hline
\end{tabular}

Source: Candidates' survey and resumes' coding. Notes : Robustness analysis for Table 8 We estimate the effects of resumes' signals on the interview rate in a model with vacant job fixed effect. Standard errors are clustered at the vacant job level. Columns 1 and 2 show returns to signals $x$ when resumes bear names (estimation of $I_{i j}=\left(1-D_{i}\right) \times X_{i} \beta^{0}(S t)+\alpha D_{i}+D_{i} \times$ $X_{i j} \beta^{1}(S t)+c_{j}+\nu_{i j}$, where $i$ indexes candidates, $j$ indexes vacant jobs and $D$ indicates minority status), and column 4 when resumes are anonymous (estimation of $I_{i j}=X_{i} \beta(A n)+c_{j}+\nu_{i j}$ ). Column 1 concerns majority candidates (results for $\beta^{0}(S t)$ ), column 2 minority candidates (results for $\alpha$ and $\beta^{1}(S t)$ ). In column 3, we report the p-value of the test of equality in returns between columns 1 and 2 . In column 5 , we report the p-value of the test of equality between columns 1, 2 and 4. For example, when nominative resumes display an interruption in labor market history of the candidate, the interview rate of majority candidates decreases by 9 points and that of minority candidates increases by 3.8 points. 